\title{
THE USE OF AN ARTIFICIAL FEMORAL HEAD FOR ARTHROPLASTY OF THE HIP JOINT
}

\author{
Jean Judet and Robert Judet, Paris, France
}

The purpose of this article is to describe a special procedure for arthroplasty of the hip. The idea of the operation, which we have called "resection-reconstruction," is excision of the pathological femoral head and its replacement by an artificial head, made of a synthetic plastic material, which is firmly fixed to the upper end of the femur. This study is based on 300 cases, the earliest of which dates back some three and a half years.

In France, up to the present time, arthroplasty of the hip has been practised mainly according to Whitman's (1924) technique (Fig. 1). Professor Mathieu in particular has taught and perfected this operation, but a review of the late results shows that out of every five cases, only one is good, one is fair, and three are poor. For a result to count as good, the patient should be able to walk for half an hour or even a full hour without pain; movement in the hip should be restored to 90 degrees of flexion and 30 degrees of abduction. In contrast, most of the patients with a poor result have almost no movement in the hip; the stiffness is commonly accompanied by an adduction deformity which advances despite the usual orthopaedic measures. Such poor results are easily explained if one considers the new mechanical conditions after Whitman's reconstruction. The femoral neck, which is about 30 millimetres in diameter, articulates with an acetabulum which is at least 50 millimetres in diameter. Furthermore, in spite of transplantation of the trochanter, the stump of the femoral neck does not always approximate to the inner wall of the acetabulum and so may remain in simple contact with the outer part of the acetabular roof in a position which favours shortening and adduction. If we operate again upon such hips we find that around the cut surface of the femoral neck there is connective tissue and new bone similar to fracture callus, together filling the acetabulum and leading to fibrous ankylosis. The retention of all the femoral neck or even of part of the femoral head, and the interposition of fascia lata or other material, reduce only slightly these imperfect results.

We thought that a more rational arthroplasty would consist in replacing the femoral head by an artificial one of the same dimensions, solidly fixed to the femoral neck. It has been our aim in this way to place between the femur and the pelvis a large and non-absorbable prosthesis and to restore stability and good function to the reconstructed joint.

\section{THE PROSTHESIS}

Design-The shape resembles that of a mushroom with a rounded head and a long stem (Fig. 2). The outer surface of the head forms two-thirds of a sphere and is highly polished. The under surface of the head is hollowed out to allow it to fit on the stump of the neck of the femur. The stem is long enough to traverse the remainder of the neck and penetrate the lateral cortex of the femur. For simplicity, the heads are made in three different sizes, each with three different lengths of stem. In this way a prosthesis for any case is always available. Materials-Rather than metal we have preferred to use an acrylic resin, methyl polymethacrylate, which is a polymer of methyl acrylic acid:<smiles>C=C(C)C(=O)O</smiles>

This plastic is not attacked by organic acids and is unaltered by a long stay in the body. 
Thermal and electrical conductivity is low. Its hardness is rated at 17.5 on the Brinell scaleabout one-quarter that of mild steel. Its resistance to shock may be expressed as follows: a thickness of 4 millimetres resists the shock of a steel ball weighing 300 grammes dropped from a height of 91 centimetres; a thickness of 8 millimetres resists the shock of a similar ball falling through 178 centimetres. With these properties it is clearly suited to be a substitute material for bone.

In twelve of our early patients the stem of the prosthesis fractured after operation. To increase strength and stiffness we therefore re-designed the prosthesis with a steel core, which is completely surrounded and insulated by the acrylic resin. The co-efficients of expansion of these two substances are almost the same at body temperatures; in addition it has been demonstrated by polarimeter measurement that the tensions developed at the steel-resin interface are insignificant. The reinforcement has quite eliminated the risk of fracture.

Tissue tolerance of methyl methacrylate - The physical and mechanical properties of a substance do not alone justify its use as an internal prosthesis; tolerance by the tissues of the body is also an essential requirement. Hitherto almost every foreign substance introduced permanently into the body has been poorly tolerated and has produced an unfavourable reaction in the

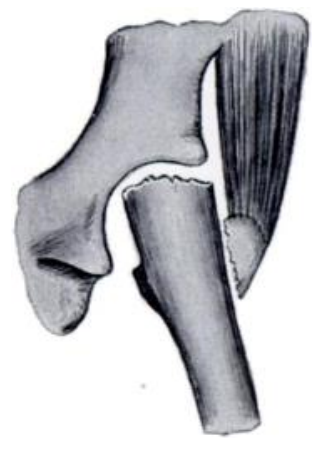

FIG. 1

Whitman reconstruction. The indifferent results of this operation in the author's hands inspired the prosthetic replacement operation. (Illustration by courtesy of Sir Reginald Watson-Jones.)

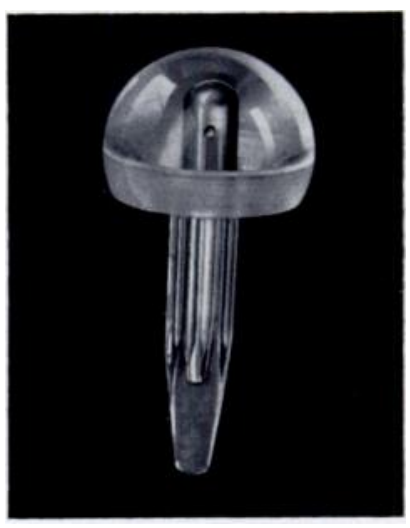

FIG. 2

The acrylic prosthesis. The stem is reinforced with a steel rod, totally enclosed within the plastic. Without this reinforcement the stem was apt to break.

surrounding tissues; "Vitallium " has for some time been a striking exception. More recently, investigations have shown that polymethacrylate is almost completely innocuous. Oto-rhino-laryngologists who have used polymethacrylate prostheses have been impressed by the absence of adhesions and of connective tissue reaction. From animal experiments, Virenque and his assistants (1947) have shown that "in muscles and in the peritoneum an acrylic resin gives rise only to a temporary reaction," and conclude that " the perfect and astonishing tolerance of acrylic resin is an established fact." Our own experience supports these conclusions. In each of the twelve early cases mentioned above, in which a second operation was necessary because the prosthesis brokc, specimens of synovial membrane, capsule and bone were taken for histological examination. Clinically no signs of inflammation were detected in any of these patients; sinus formation did not occur even when part of the broken prosthesis became superficial and without muscle cover. The tissues removed at operation showed little variation from their normal structure.

From the radiological aspect, a somewhat inconvenient property of polymethacrylate is its translucency to X-rays. On the other hand this allows complete examination of the structure of the bone in contact with the prosthesis. In 150 cases observed over various

vol. $32 \mathrm{~B}$, NO. 2 , MAY 1950 
periods between six months and three and a half years, no osteoporosis of bone has been visible in the radiographs. This record, and the fact that none of the stems has loosened, lead us to expect that the sound fixation of the prosthesis in the femur secured at operation will be maintained indefinitely. The failures that have been reported with other materials (Robineau 1926) were due to a rarefying osteitis which was caused by these materials and which allowed the prosthesis to become loose in the femoral neck.

\section{OPERATIVE TECHNIQUE}

The exposure-In order to obtain good movement later we believe that it is essential to avoid all damage to muscle and bone. We therefore use Huter's vertical incision, which extends about 15 centimetres down from the anterior-superior iliac spine, passes between tensor fasciae latae and sartorius, then lateral to rectus femoris and down to the capsule. Branches of the anterior circumflex artery are usually found in the lower angle of the wound and may need to be ligated. The capsule is freely exposed over its anterior, superior and inferior aspects with a blunt instrument.

Excision or incision of the capsule-The capsule is always pathological and adherent to the head and neck of the femur. When grossly abnormal it is resected; in other cases we prefer to make a cruciate incision, which allows dislocation of the head. The capsule having been freely opened, a retractor is slid round each border of the neck to give full exposure of the head and acetabulum.

Resection of the head-Two methods may be used. When the acetabulum is shallow and the head tends to subluxate, dislocation is completed and the head resected with a chisel. When the acetabulum is deep the neck is divided at its junction with the head, and the head extracted with Mathien's hook and Lambotte's spoon. Any residual osteophytes are excised, particularly those so frequently found near the lower margin of the femoral head in osteoarthritis. Treatment of the acetabulum-Inspection of the acetabulum is made easier by medial rotation and slight flexion of the femur. If the roof is oblique, or if the lower part is more or less filled with bone, the necessary amount is hollowed out with a simple reamer driven by hand (Fig. 3b) or by an electric motor. The reamers we have designed are similar in shape to the plastic heads, though the diameters are slightly larger. It is preferable that the head should have free play in the acetabulum and not fit too snugly. In cases of chronic arthritis, such hollowing out of the acetabulum is necessary in two-thirds of the cases.

Drilling and preparation of the neck-The femoral neck is traversed with a drill one millimetre less in diameter than the stem of the prosthesis-a point of vital importance. The lower cortex of the femoral neck is a useful guide. The outer cortex of the femur is perforated, and the length of the tunnel indicates the appropriate length of stem. The common dimensions are 6,7 and 8 centimetres.

In order that the hollowed-out under-surface of the plastic head should fit the neck closely at the point of section and derive some support from it, the end of the neck is modelled with a special cutter centred on the axial tunnel (Fig. 3a). The stem of the prosthesis is now gently hammered into the bony tunnel till the head is well seated on the neck of the femur. An aluminium punch with a large concave head (Fig. 3d) is used to avoid damaging the highly polished surface of the prosthesis. Should the bevelled end of the stem project much beyond the cortex of the femur, a small incision may be needed in order to trim the acrylic tip. On no account should the length of stem be so much in excess that the metal reinforcement also projects, as the steel rod cannot be cut close to the bone.

To reduce the new femoral head into the acetabulum, traction is applied to the leg, which is then rotated inwards and abducted. A Murphy's skid is sometimes helpful. If the capsule has not been resected, it is repaired with a few catgut sutures. The muscles fall into position, and the aponeurosis is sutured with care that the lateral cutaneous nerve of the thigh is not included. 
Post-operatice treatment-Previously it was our practice to immobilise the hip in plaster for three weeks. More recently a simple suspension frame has been used, and mobilisation begun between the tenth and twelfth days. The apparatus allows four movements which the patient can control by means of ropes-elevation of the whole limb, flexion of the knee alone, simultaneous flexion of the knee and hip and, finally, abduction and adduction. Massage is started at the same time and after three weeks the patient begins to walk, at first with the help of a nurse, then alone with two sticks and soon with a single stick.

\section{RESULTS}

The rate of recovery of function is very variable. Age, muscle tone, the regularity of the post-operative exercises and the will-power of the patient are important factors. In half the cases the range of movement after two or three months is excellent, and light work may be resumed. In other cases recovery is slower and partial incapacity lasts six to eight months.

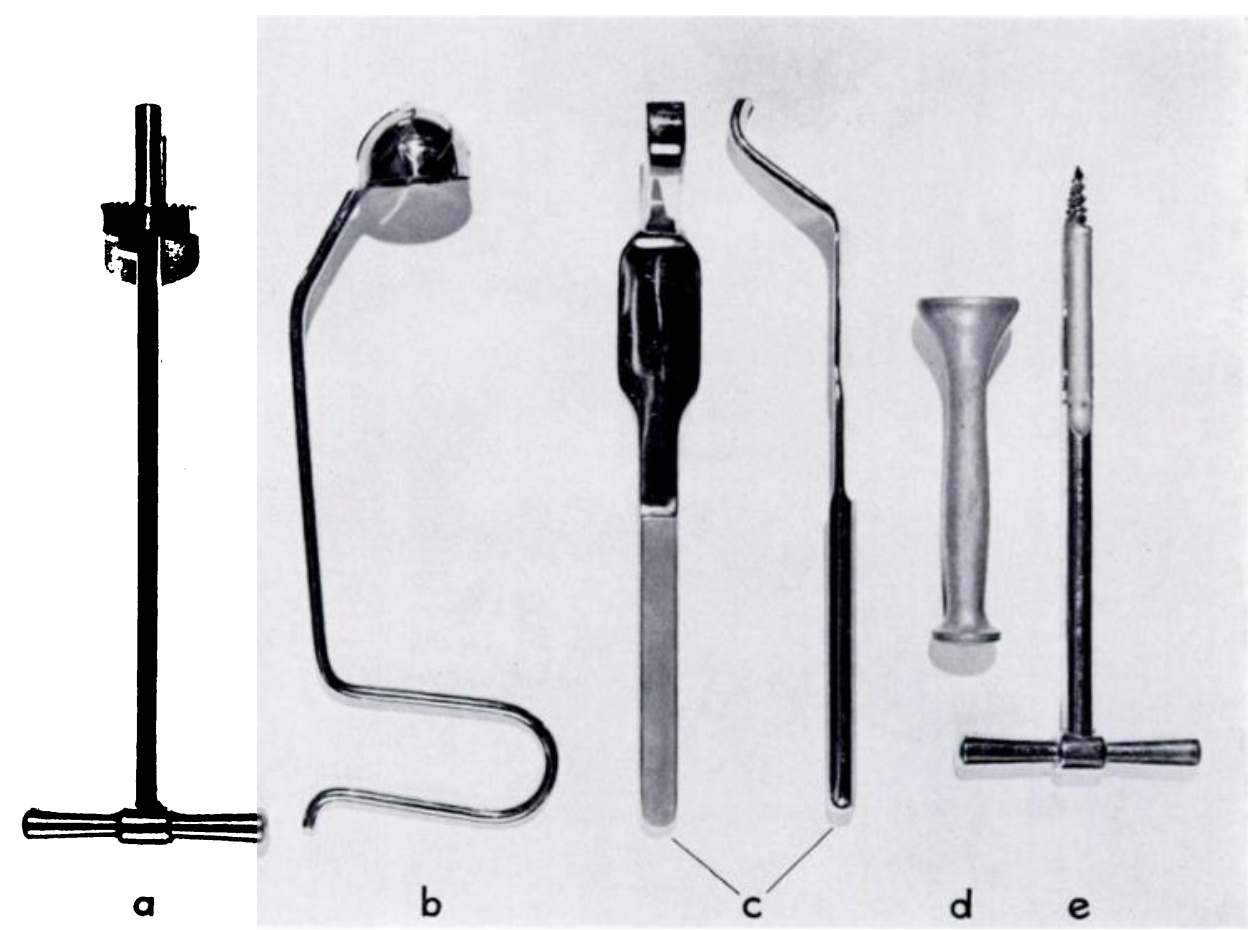

FIG. 3

Some of the instruments used in the operation: (a) cutter for shaping femoral neck, (b) reamer for acetabulum, $(c)$ two aspects of a retractor for neck of femur, (d) concave-headed impactor for driving prosthesis into femoral neck, $(e)$ hand drill for femoral neck.

Pain-In no case has the pain been made worse by operation. Seven patients out of ten have either no pain or a negligible amount. This freedom from pain is quite remarkable when one remembers that intensity of pain is the main indication for operation. Patients with bilateral arthritis have often requested early operation on the other side.

Three patients out of ten still have pain, which is, however, moderate in degree and tolerable. The pain is usually referred to the lateral or anterior surface of the hip; it may be felt after lying down or sitting and may diminish after a few minutes of activity. Local infiltration of novocain and, occasionally, radiotherapy may give some relief. Even in these less satisfactory cases walking and other activities can be sustained for longer periods than previously.

VOL. $32 \mathrm{~B}$, พO. 2 , MAY 1950 


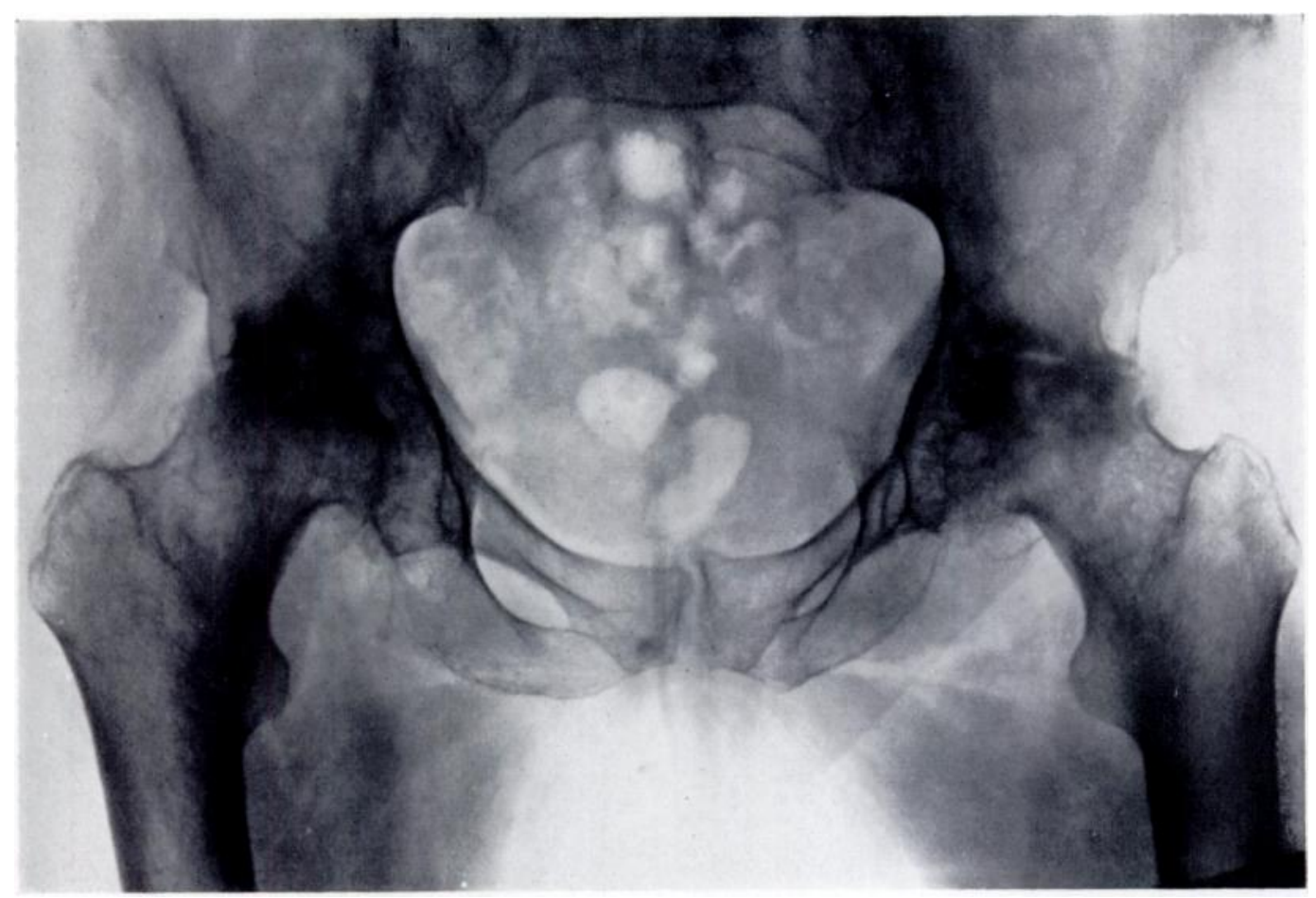

Fig. 4

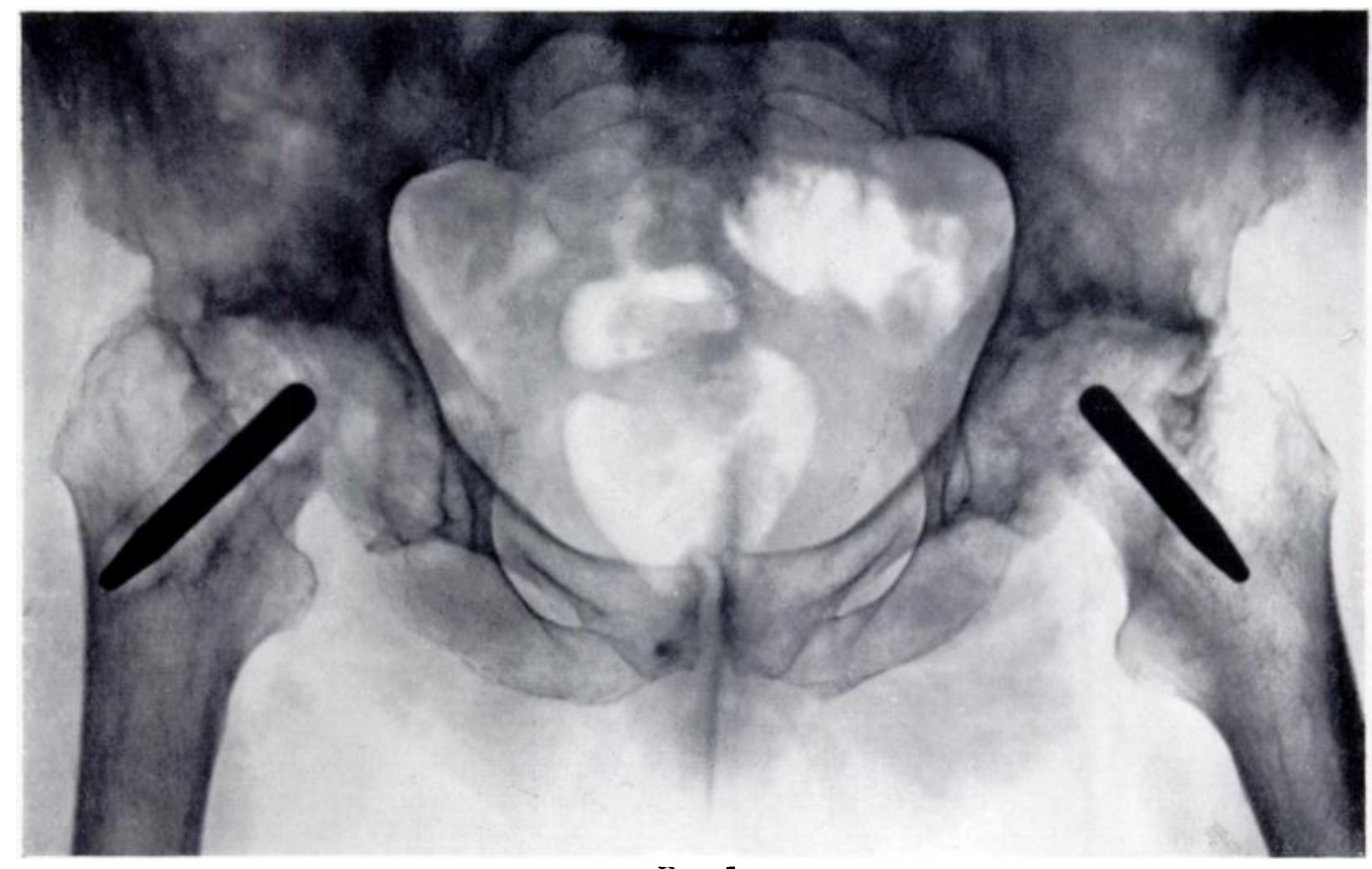

FIG. 5

Case 1. Female aged seventy-two years. Bilateral osteoarthritis. Figure 4--Condition before operation. Figure 5-Condition after excision of femoral heads and replacement with acrylic prostheses. 


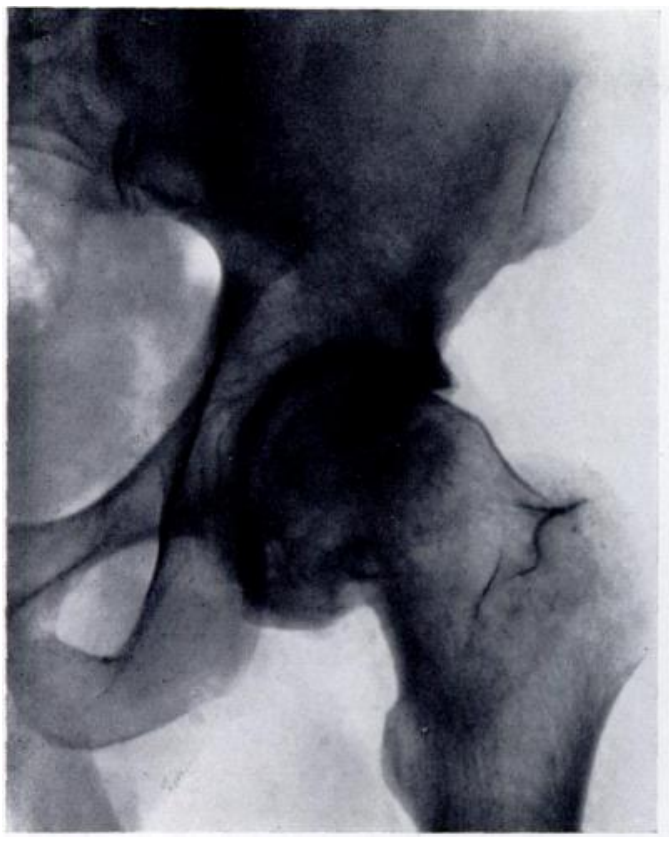

FIg. 6

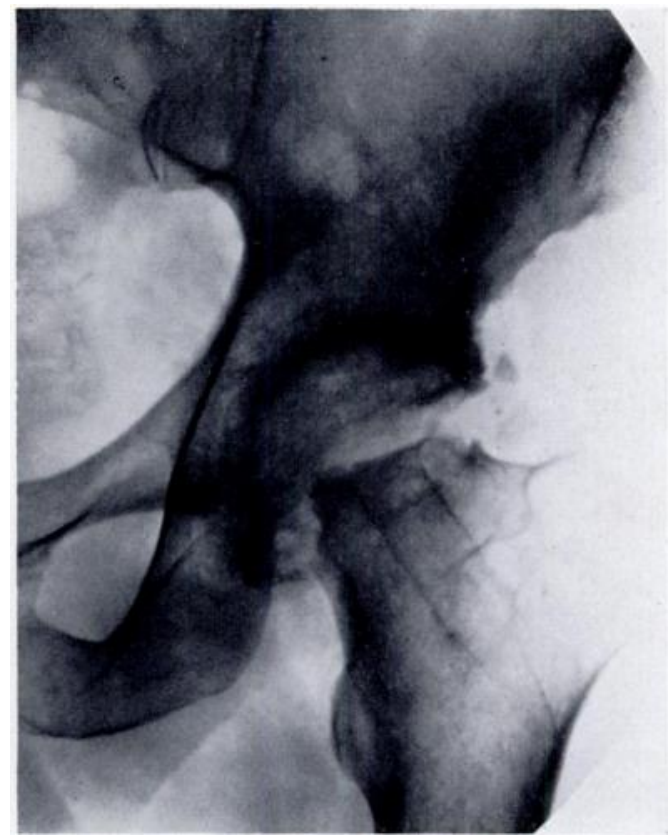

FIG. 7

Case 2. Female, aged fifty-seven years. Osteoarthritis. Figure 6-Before operation. Figure 7-Two and a half years after operation. An early-type prosthesis, without metal reinforcement of stem, was used. Position of stem is indicated by condensation of surrounding bone.

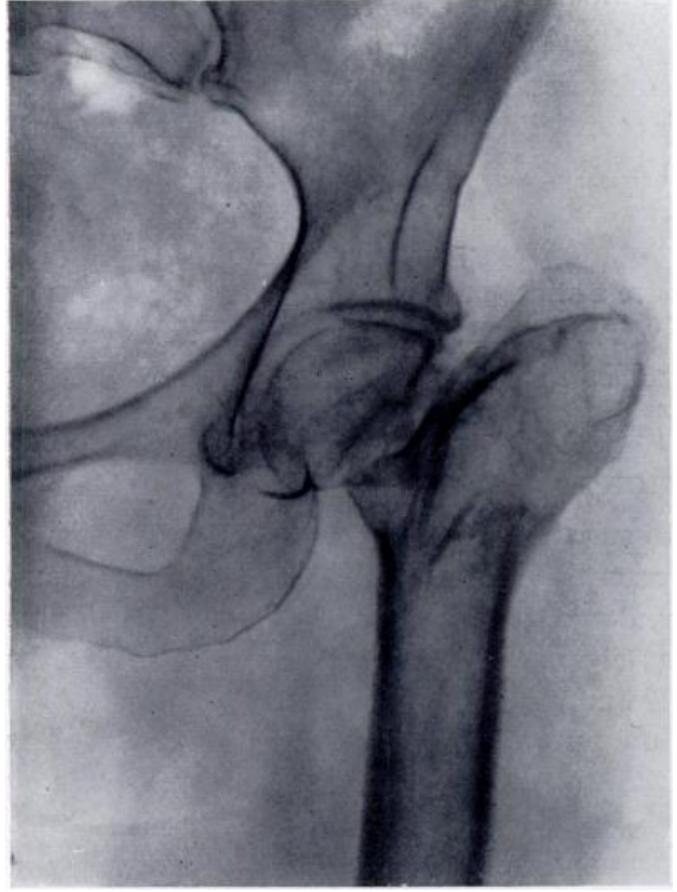

Fig. 8

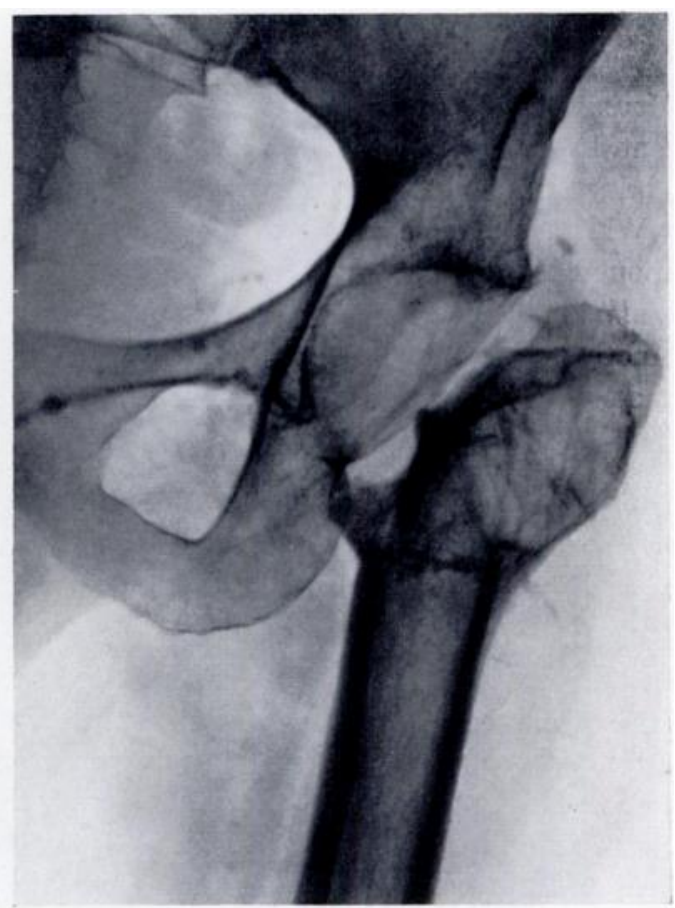

Fig. 9

Case 3. Ununited fracture of femoral neck. Subtrochanteric osteotomy was unsuccessful in relieving symptoms. Painful pseudarthrosis (Fig. 8). The head was excised and replaced by an acrylic prosthesis. The track of the stem can just be seen in Figure 9.

VOL. $32 \mathrm{~B}$, No. 2, MAY 1950 


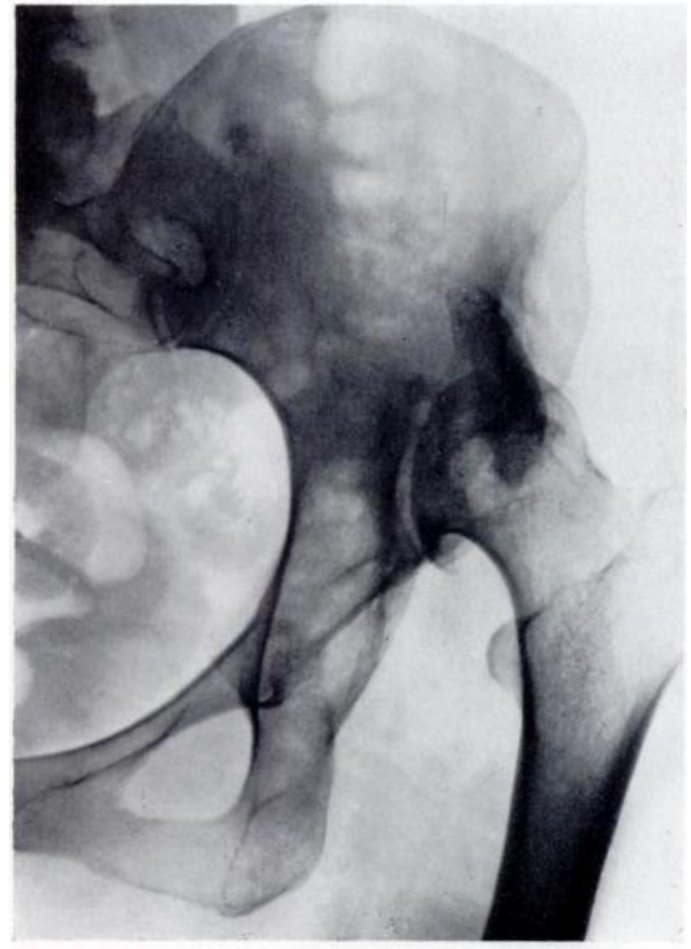

Fig. 10

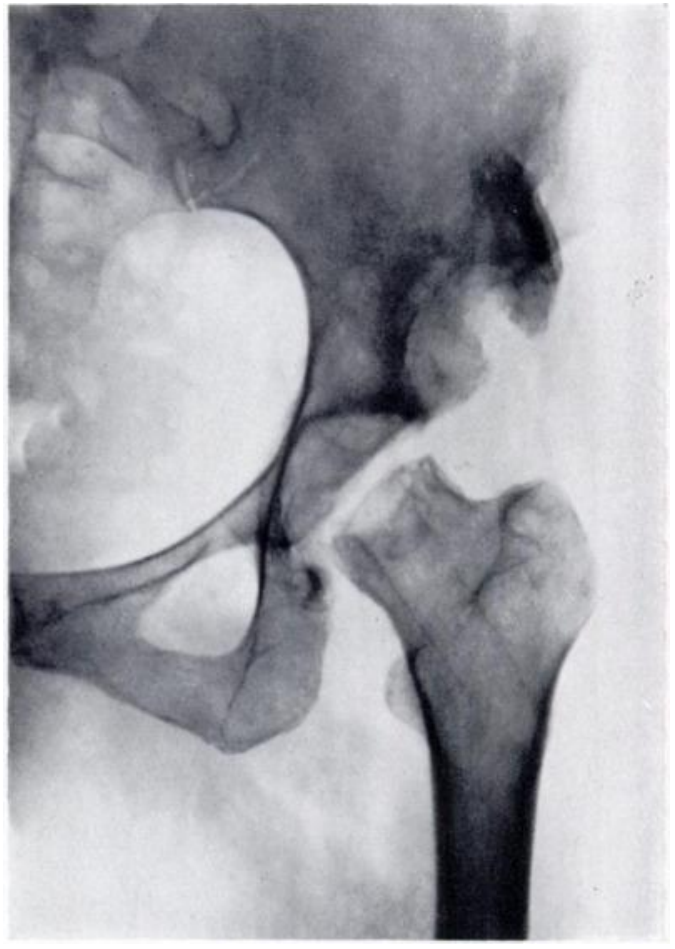

FIG. 11

(ase 4. Female, aged twenty-six years. Congenital dislocation of left hip. Unsuccessful shelf operation. Note marked upward displacement of femoral head (Fig. 10). A new acetabulum was formed in normal position; the head was excised and replaced with an acrylic prosthesis (Fig. 11).

Morement-With regard to flexion, 60 per cent. of patients reach or pass 90 degrees; 30 per cent. have from 60 to 90 degrees; and only 10 per cent. fail to obtain 60 degrees of movement. Abduction is never less than 20 degrees, and the fixed adduction deformity so frequent after a Whitman's resection has not been seen. The range of medial and lateral rotation is about half the normal.

Walking-One-third of the patients do not require a stick, but two-thirds do. The return of function is variable but often remarkable; 80 per cent. of patients are able to return to work, and some can walk several miles, ride a bicycle and do a heavy day's work.

\section{INDICATIONS}

The " resection-reconstruction" operation is suitable for the treatment of four types of lesion: osteoarthritis, ununited fracture of the femoral neck, non-tuberculous ankylosis and dislocation of the hip. Patients of any age can undergo this operation; we judge their fitness on the general state of health and on the results of routine laboratory tests. Three deaths have occurred after 300 operations, possibly from improper selection. One patient of seventy-three years died on the third day, but the autopsy revealed no special cause. The second death was caused by uraemia during the third week, and the last by cerebral softening in the serenth week.

Ostcoarthritis (Figs. 4 to 7 )-This is much the commonest condition requiring operation, and in these cases the principal indication is pain that has failed to respond to conservative treatment. Operation is reserved for those whose activities by day and rest by night are badly disturbed, though an adduction contracture or bilateral fibrous ankylosis are secondary indications. 
THE USE OF AN ARTIFICIAL FEMORAL HEAD FOR ARTHROPLASTY OF THE HIP JOINT

Ununited fracture of the femoral neck (Figs. 8 and 9)-This is the second most important indication. So far, we have operated on twenty of these cases with such success that we consider the treatment to have no equal for ease and speed. For special reasons we have twice treated recent fractures in the same way, and again both gait and range of movement have been excellent. We must add that after non-union the neck is often decalcified or reduced to a small stump; the great trochanter should then be displaced by Whitman's procedure in order to give lengthening of the neck. The degree of upward displacement of the femur is of no importance.

Non-tuberculous ankylosis of the hip-In such cases the femoral neck is divided with a curved chisel close to the ilium, which is then hollowed out in order to form a new acetabulum. The operation is successful when muscle power is adequate.

Dislocation of the hip (Figs. 10 and 11)-For an old congenital dislocation with pain, the original acetabulum is deepened and the head of the prosthesis reduced into it. Sometimes a new acetabulum has to be created at a higher level. The operation is suitable also for cases of dislocation after infective arthritis of infancy when the head has already been destroyed.

\section{CONCLUSIONS}

We have endeavoured to make known the results of a simple type of arthroplasty of the hip which is well tolerated even by elderly patients. It has not been the purpose of this article to compare former operations with our own, a newcomer in the field of surgery of the hip. In particular we do not wish to compare our comparatively recent results with Smith-Petersen's well-established method of cup arthroplasty-a magnificent contribution to the surgery of the region. The results we have already obtained, however, appear sufficiently favourable to merit further study and development of the method. If the future confirms the value of our early results, we shall perhaps be able to suggest operation to patients who suffer more from loss of function than from pain.

\section{REFERENCES}

Robineau, - (1926) : Prothèse osseuse perdue en metal à revêtement d'ébonite. Journal de Chirurgic, 27, 51. Virengue, M., Leroux, R., Delaunay, A., Delaunay, M., Lasfargues, E., Coronel, S., and Kerneis, J. P. (1947): Les résines acrỵliques en prothèse et en biologie. Presse Médicale, 55, 736.

Whitman, R. (1924): The reconstruction operation for arthritis deformans of the hip joint. Annals of Surgery, 80, 779 .

VOL. 32 B, No. 2 , MAY 195 () 